\title{
Spontaneous Bilateral Simultaneous Tubal Pregnancy
}

\author{
Vyas NM, ${ }^{1}$ Manjeera $L,{ }^{1}$ Shetty $D,{ }^{1}$ Rai $S^{1}$ \\ ${ }^{1}$ Department of Obstetrics and Gynaecology, KS Hegde Medical Academy, Deralakatte, Mangalore, Karnataka, India.
}

\begin{abstract}
Bilateral tubal pregnancy in absence of ovulation induction represents the rarest form of ectopic pregnancies. Among the variant of twin ectopic pregnancy, heterotopic or twin pregnancy in the same tube is higher compared to that of bilateral tubal pregnancy. Till date around 250 cases of simultaneous bilateral tubal pregnancy have been detected and many occur following assisted reproductive treatment. Preoperative diagnosis is uncommon. It is usually detected intraoperatively and so necessitates careful examination of both the adnexa. We report a case where in one tube showed ruptured tubal pregnancy and in the other tube, a mass was noticed which was suspected to be a hydrosalphnix or a pyosalphinx. Since patient had already consented for tubectomy, a bilateral salphingectomy was done. Histopathology showed bilateral tubal pregnancy.
\end{abstract}

Keywords: pregnancy, ovulation induction, tubal pregnancy.

\section{INTRODUCTION}

The incidence of bilateral ectopic pregnancy is $1 / 725$ to $1 / 1580$ of all ectopic pregnancies and is around $1 / 2,00,000$ pregnancies. ${ }^{1}$ The incidence has increased in the recent past after the introduction of assisted reproductive treatment, increased use of antibiotics for pelvic inflammatory disease and increased rate of tubal surgeries. Though the occurrence of bilateral tubal and heterotopic pregnancy is more with assisted reproductive treatment, the incidence of spontaneous bilateral ectopic is exceedingly rare. Simultaneous bilateral ectopic pregnancy is least common type of all the ectopic implantation of two embryos.

\section{CASE}

A lady aged 40 years presented to emergency with severe pain abdomen. She was G4 P2 L1 A1 with seven weeks of amenorrhoea and her urine pregnancy test was positive. She had no vaginal bleeding or discharge. In her first pregnancy, she had an intrauterine death at eight months of amenorrhoea. She underwent a caesarean section in her second pregnancy and has a 10 year old child. Three years back she underwent curettage for incomplete abortion. She had no past history suggestive of pelvic inflammatory

\section{CORRESPONDENCE}

Dr Neetha Vyas

Department of Obstetrics and Gynaecology,

KS Hegde Medical Academy, Karnataka, India.

Email: nvyas_21@yahoo.com

Phone: +919900001287 disease. This spontaneous conception was not a planned one. On examination, her pulse rate was 90 beats per minute and blood pressure was $100 / 60 \mathrm{mmHg}$. Abdominal palpation revealed left iliac tenderness without guarding or rigidity. Bimanual examination revealed left fornix mass and tenderness with normal sized uterus. Ultrasonography showed empty uterine cavity, a live fetus of 7 weeks 4 days in the left adnexa and with significant free fluid in peritoneal cavity (Figure 1).

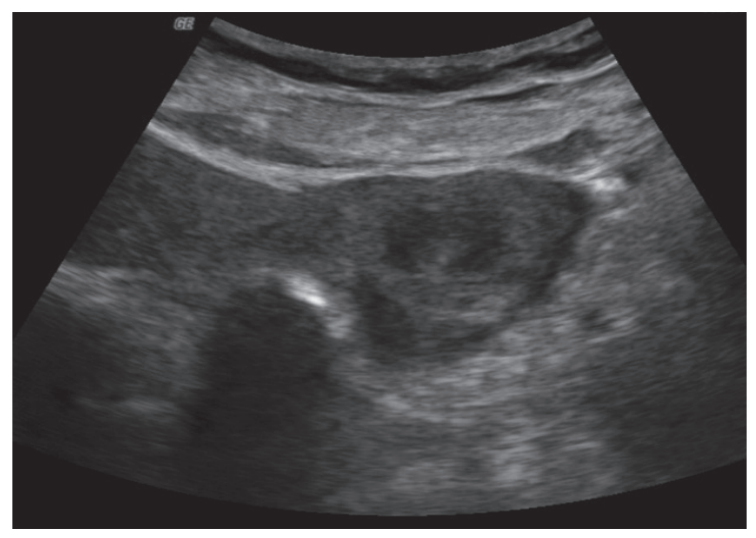

Figure 1. Ultrasonography showing left sided live ectopic pregnancy with free fluid in the peritoneum.

The papers in this journal are published under the terms of the Creative Commons Attribution License. Users are allowed to read, download, copy, distribute, print, search, or link to the full texts of the articles in this journal without asking prior permission from the publisher or the author. 
An emergency laporotomy was done. Patient had consented for tubectomy too. Intraoperatively, there was $200 \mathrm{ml}$ of hemoperitoneum. In the left fallopian tube, a $4 \mathrm{x}^{4}$ $\mathrm{cm}$ mass was found at fimbrial end with bleeding from the site. With gentle manipulation of the tube, the entire intact conceptus expelled out from the rent in the fallopian tube (Figure 2). Right tube also showed a mass of $2 \times 1 \mathrm{~cm}$ at the fimbrial end. Hydrosalphinx or pyosalphinx was suspected. Bilateral salphingectomy was done. Postoperative period was unremarkable.

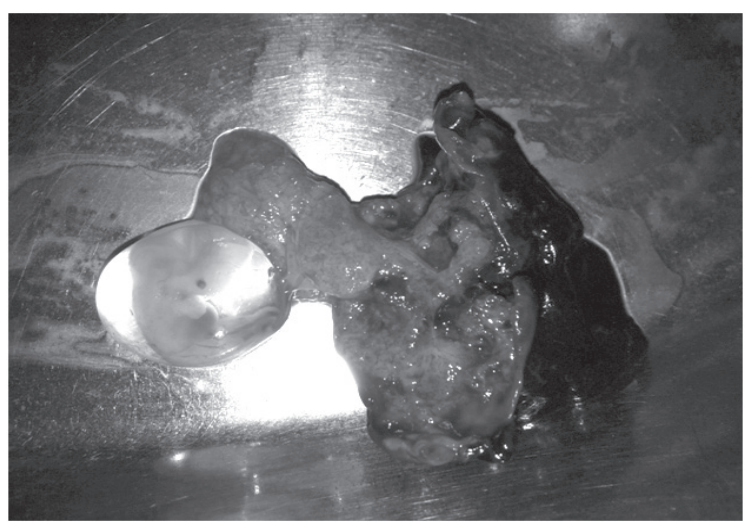

Figure 2. The conceptus which was expelled from the ruptured left fallopian tube.

Histopathology revealed synctiotrophoblast and cytotrophoblast, chorionic villi and decidualization in both fallopian tubes with fetal parts in the left.

\section{COMMENT}

The clinical presentation of bilateral tubal pregnancy is no different from unilateral tubal pregnancy. One needs to have a high level of suspicion to diagnose bilateral ectopic pregnancy. The preoperative diagnosis of bilateral tubal pregnancy is very uncommon. Ultrasonography cannot be relied upon to make the diagnosis of bilateral tubal pregnancy. A case has been reported where in a preoperative diagnosis of bilateral tubal pregnancy was done by ultrasonography. ${ }^{2}$ The diagnosis is usually made intraoperatively. So, it is always recommended to closely examine the other tube thoroughly. Even in the present case, the contralateral tubal mass was not suspected to be an ectopic pregnancy.

In 1939, Fishback suggested presence of fetuses or any portion of them along with placental material was the criteria for simultaneous bilateral tubal pregnancy. ${ }^{3}$ But in 1953, Norris modified this criteria stating presence of chorionic villi in each tube should suffice. ${ }^{4}$
There is no separate guideline for treatment of synchronous tubal pregnancy because of its' rarity. But the principles of treatment of unilateral ectopic pregnancy can be applied here. ${ }^{5}$ Currently, laparoscopy is the choice for elective management. Firstly, it is useful in diagnosing bilateral tubal pregnancy which could have been missed by ultrasonography. As in the case reported by Sommer et $\mathrm{al}^{6}$ while administering methotrexate under laparoscopy guidance, bilateral tubal pregnancy was diagnosed and bilateral salphingostomy stopping methotrexate. In case where the tube is destroyed salphingectomy is done. In case of intact tube, salphingostomy is preferred. But the patient has to be counseled regarding the risk of recurrent ectopic and need for another surgery. Follow up with serum human chorionic gonadotrophin is necessary to rule out persistent trophoblastic disease if the tubes are conserved. Another case reported by Ryan et al, ${ }^{7}$ with a ruptured tubal pregnancy and 'clubbed' appearance in the other tube, interpreted as residual scarring from the previous ectopic pregnancy presented ten days later with ruptured tubal pregnancy on the side of scarred tube. The need of high index of suspicion has been stressed and probably a follow up with human chorionic gonadotrophin could have been useful in early detection. A patient who has had one ectopic pregnancy is at risk of having another ectopic not just in future pregnancy, but also at the same time. Vigilance and early detection of unusal ectopic pregnancy can avoid potential fatal complications and morbidities.

\section{REFERENCES}

1. Adair CD, Benrubi GI, Ramos S, Rhatigan R. Bilateral tubal ectopic pregnancies after bilateral partial salpingectomy: a case report. J Reprod Med. 1994;39(2):131-3.

2. Martinez J, Cabistany AC, Gonzalez M, Gil O, Farrer M, Romero JA. Bilateral simultaneous ectopic pregnancy. South Med J. 2009;102(10):1055-7.

3. Fishback HR. Bilateral simultaneous ectopic pregnancy. Am J Obstet Gynecol. 1939;37:1035.

4. Norris S. Bilateral simultaneous tubal pregnancy. Can Med Assoc J. 1953;68:379-81.

5. Kelly AJ, Sowter MC, Trinder J. The management of tubal pregnancy. London: RCOG Press; 2004.

6. Sommer EM, Reisenberg K, Bogner G, Nagele F. Laparoscopic management of an unrecognized spontaneous bilateral tubal pregnancy. Acta Obstet Gynecol Scand. 2002;81(4):366-8.

7. Ryan MT, Saldana B. Bilateral tubal ectopic pregnancy: a tale of caution. Acad Emerg Med. 2000;7(10):1160-3. 